Revista de Iniciação Científica em Odontologia. 2018;16(3):129-135

ISSN 1677-3527 - DOI: 10.4034/RevICO.2018.16.3.13

Relato de Caso

\title{
Tratamento de Fístula Buco-sinusal: proposta de abordagem
}

\author{
Treatment of oroantral fistula: proposal of surgical approach
}

Yuri Barbosa Alves; Ávilla Pessoa Aguiar²; Fábio Correia Sampaio³; Isadora Serrão Wanderley; José Wilson Noleto ${ }^{4 ;}$ Thalles Moreira Suassuna ${ }^{5}$

${ }^{1}$ Acadêmico de Odontologia da Universidade Federal da Paraíba, João Pessoa - Paraíba - Brasil ${ }^{2}$ Acadêmica de Odontologia no Instituto de Educação Superior da Paraíba, João Pessoa Paraíba - Brasil

${ }^{3}$ Professor de Odontologia da Universidade Federal da Paraíba, João Pessoa - Paraíba - Brasil ${ }^{4}$ Professor de Cirurgia e Traumatologia Buco-Maxilo-Facial da Universidade Federal da Paraíba, João Pessoa - Paraíba - Brasil

${ }^{5}$ Mestrando em Odontologia da Universidade Federal da Paraíba, João Pessoa - Paraíba - Brasil

Autor e endereço para correspondência:

José Wilson Noleto - Universidade Federal da Paraíba, Centro de Ciências da Saúde - Campus I, Departamento de Odontologia Restauradora. João Pessoa, PB - Brasil - CEP: 58051900. Email: wilsonnoleto@ig.com.br

\section{Resumo}

Introdução: A comunicação buco-sinusal compreende uma abertura criada entre o seio maxilar e a cavidade oral, que, se não tratada, evolui para uma Fístula BucoSinusal (FBS). Apresenta inúmeros fatores etiológicos, sendo o trauma e acidentes durante exodontias os mais frequentes. Tal condição permite a passagem de fluidos e bactérias para o seio maxilar e predispõe a sinusite maxilar. $O$ tratamento cirúrgico através da confecção de retalhos pediculados muitas vezes é indicado. A escolha da técnica específica a ser empregada levará em consideração a região da comunicação ou fístula, anatomia local e a experiência do cirurgião. Relato de caso: Paciente CPS, 42 anos, gênero feminino, à primeira consulta apresentava infecção aguda no seio maxilar com secreção purulenta. Queixava-se ainda de dor em hemiface, disosmia e voz anasalada. Clinicamente observou-se uma pequena fenestração em processo alveolar totalmente edêntulo, por onde saía a secreção. Foram instituídos antibióticos, irrigações com soro e tratamento cirúrgico para remoção da fístula e fechamento da comunicação. Foi realizado o retalho bucal com o uso do corpo adiposo para fechamento em duas camadas. Após 2 meses observamos boa cicatrização e obliteração da comunicação. Conclusão: Conclui-se que as comunicações bucosinusais são condições que podem comprometer a saúde do indivíduo e merecem tratamento cirúrgico. Dentre uma diversidade de técnicas, o retalho bucal com corpo adiposo se apresenta como uma opção prática, efetiva, segura, de baixo custo e com bons resultados.

Descritores: Fístula buco-sinusal. Cirurgia. Corpo Adiposo Bucal. 


\begin{abstract}
Introduction: Oroantral communication is an opening created between the maxillary sinus and the oral cavity, which, if untreated, evolves into a oroantral fistula. It presents numerous etiological factors, being the trauma and accidents during exodontia the most frequent. This condition allows the application of fluids and bacteria to the maxillary sinus and predisposes to maxillary sinusitis. Surgical treatment through the manufacture of pedicled flaps is often indicated. The choice of the specific technique to be employed will take into account the region of communication or fistula, local anatomy, and the surgeon's experience. Case report: Patient CPS, 42 years old, female, at the first visit had acute infection in the maxillary sinus with purulent secretion. She still complained of pain in hemiface, dysosmia and nasal voice. Clinically it was observed a small fenestration in a totally edentulous alveolar process, through which secretion exited. Antibiotics, irrigations with serum and surgical treatment were instituted to remove the fistula and to close the communication. The buccal flap was performed using the buccal fat pad for closure in two layers. After 2 months we observed good cicatrization and obliteration of the communication. Conclusion: It is concluded that oroantral communications are conditions that can compromise the health of the individual and deserve surgical treatment. Among a variety of techniques, buccal flap with adipose body presents itself as a practical, effective, safe, low-cost and successful option.
\end{abstract}

Keywords: Oroantral Fistula. Surgery. Buccal Fat Pad.

\title{
Introdução
}

A comunicação buco-sinusal é a abertura patológica criada entre o seio maxilar e a cavidade oral, que, se não tratada, evolui para uma Fístula Buco-Sinusal (FBS)1. Tal condição geralmente se desenvolve como uma complicação após a extração dos dentes molares superiores e é mais provável que aconteça quando um dente possui raízes muito próximas do seio maxilar ou até dentro do seio².

A execução de uma técnica cirúrgica agressiva e excesso de curetagem alveolar pósextração também facilitam sua ocorrência. Outras causas mais comuns da comunicação bucosinusal são a destruição da parede do seio por cistos ou tumores benignos ou malignos, cirurgia de implante, osteomielite ou trauma com perda de substância ${ }^{3}$.

Os principais sinais clínicos da comunicação são o teste de Valsava positivo, a passagem de líquidos da boca para o nariz, voz anasalada, sinusite maxilar recorrente e a sensação de cheiro ruim (disosmia). Radiograficamente pode ser visualizado um defeito ósseo na região e um espessamento da mucosa sinusal ${ }^{4}$. 
As técnicas cirúrgicas empregadas para o fechamento das comunicações vão desde o fechamento primário do alvéolo durante a exodontia até os retalhos pediculados, que podem ser palatinos ou vestibulares (utilizando ou não o corpo adiposo bucal e biomateriais) ${ }^{1,3,4}$.

\section{Relato de Caso}

Paciente CPS, 42 anos, gênero feminino e com história de exodontia traumática há cerca de 5 anos. Queixava-se de fortes dores oriundas do seio maxilar, disosmia e voz anasalada. Relatou episódios esporádicos de passagem de líquidos da boca para o nariz e que já tinha se submetido a procedimento cirúrgico corretivo na região.

Clinicamente observou-se rebordo alveolar superior esquerdo totalmente edêntulo, área central de hiperemia (sensível à palpação) e passagem de secreção purulenta por pequeno orifício entre a mucosa alveolar e mucosa do palato. Ao exame de imagem (radiografia panorâmica) constatou-se um defeito ósseo importante ao longo do rebordo alveolar medindo cerca de $2 \mathrm{~cm}$ no maior diâmetro.

A conduta adotada foi irrigação abundante da fistula para drenagem de todo o conteúdo purulento e prescrição de amoxicilina com clavulonato de potássio durante 10 dias. A paciente foi orientada a realizar irrigações com soro fisiológico 0,9\% em casa, utilizando seringa e agulha sem bisel, sendo iniciadas 7 dias antes da cirurgia. Após remissão do quadro infeccioso local, foi instituído tratamento cirúrgico através de fistulectomia para remoção de todo o trato fistuloso, sinusectomia maxilar para remoção da membrana infectada do seio e avanço de retalho bucal para fechamento da comunicação. O fechamento aconteceu em dois planos.

Após a sinusectomia e fistulectomia foi realizada uma divisão do retalho (para melhorar a distensibilidade dos tecidos), identificação do Corpo Adiposo Bucal e divulsão ao redor do mesmo para utilizá-lo no fechamento. O tecido adiposo bucal foi então suturado nas bordas da ferida operatória e em seguida recoberto pela mucosa, a qual foi suturado sem tensão.

A paciente apresentou boa evolução, sem complicações, com resolução das suas queixas e exibindo boa cicatrização dos tecidos moles, sendo proservada durante 6 meses. 

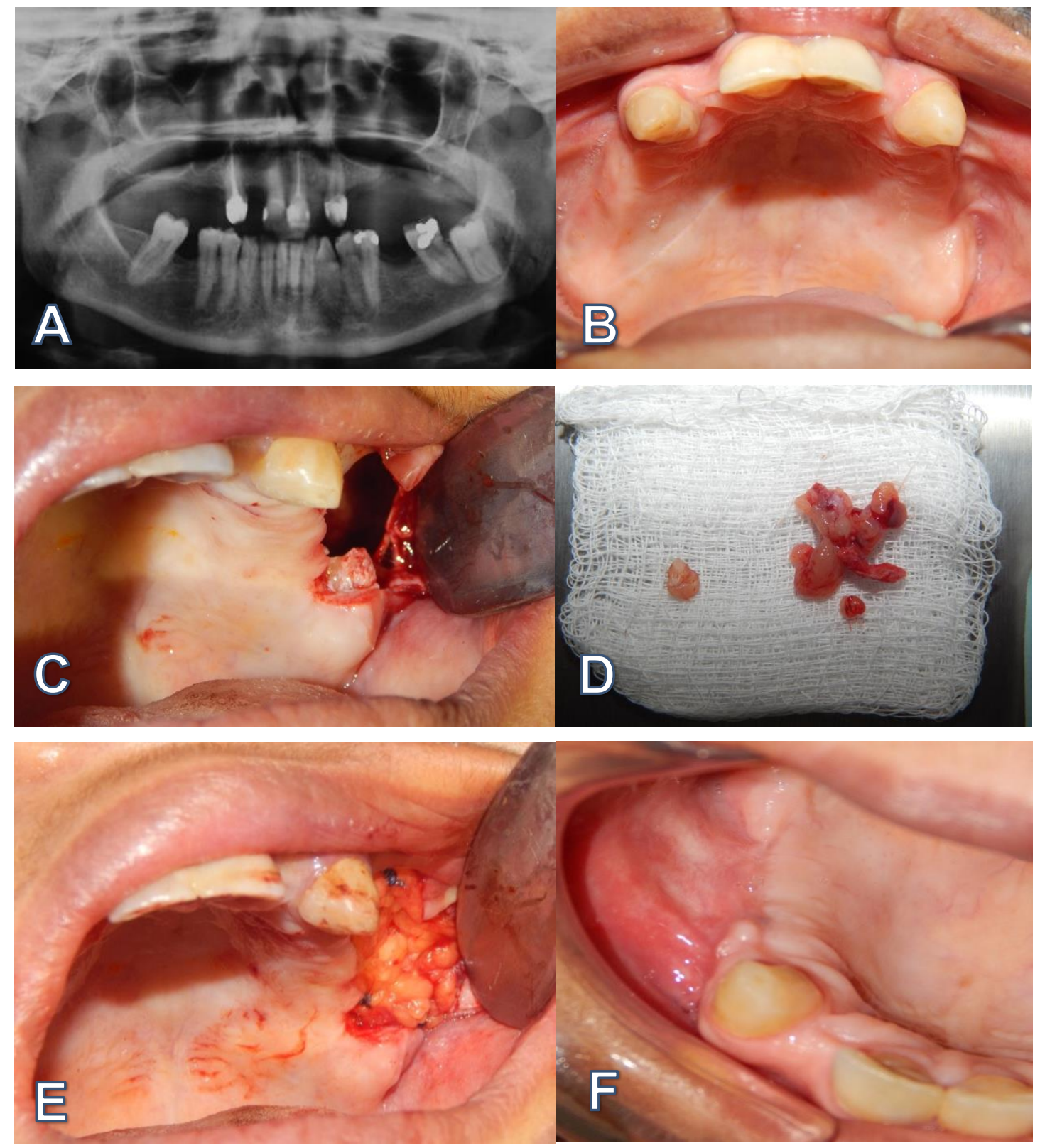

A) Radiografia Panorâmica inicial, evidenciando defeito ósseo importante ao longo do rebordo alveolar superior esquerdo medindo cerca de $2 \mathrm{~cm}$ no maior diâmetro. B) Aspecto intra-oral evidenciando saída da fístula bem discreta e sem inflamação no momento do registro. C) Acesso para a fistulectomia. Observe a extensão do defeito ósseo. D) Membrana mucosa do seio maxilar após remoção bastante espessada. E) Fechamento da comunicação com o tecido adioposo bucal em primeiro plano. F) Pós-operatório de 2 meses exibindo boa cicatrização. 


\section{Discussão}

A comunicação buco-sinusal e a consequente fístula são complicações comuns após a extração dentária dos molares superiores, sendo esta a causa mais frequente, representando cerca de $92,63 \%$ dos $\operatorname{casos}^{2,5,6}$.

O fechamento de uma comunicação é muito importante para evitar a fuga de comida e saliva para o seio maxilar, evitando assim a contaminação do seio com bactérias provenientes da cavidade bucal ${ }^{5}$. Geralmente defeitos menores que $3 \mathrm{~mm}$ de diâmetro e sem epitelização se curam espontaneamente. No entanto, a intervenção cirúrgica é necessária para o fechamento em diâmetros maiores que 3 a $5 \mathrm{~mm}^{5-7}$.

Várias técnicas cirúrgicas são sugeridas para o fechamento das comunicações e na escolha da técnica vários fatores devem ser considerados, tais como: a localização e tamanho do defeito, a duração da fístula, a presença de infecção sinusal, a disponibilidade de recursos e a saúde geral do paciente ${ }^{7-9}$.

Classicamente as técnicas mais utilizadas no fechamento das comunicações e fístulas são o retalho bucal (podendo usar ou não o corpo adiposo bucal) e o retalho palatino. Recentemente vêm sendo descritas técnicas que empregam materiais aloplásticos como barreiras mecânicas no fechamento, bem como membranas de Fibrina Rica em Plaquetas (PRF). No entanto, devido ao alto custo, estas opções encontram dificuldade em se difundir. Além disso, ainda são necessários mais estudos para validar seus resultados no tratamento das $\mathrm{FBS}^{8,10,11}$.

A técnica de retalho bucal apresenta uma taxa de sucesso variando de $84 \%$ a $93 \%$, enquanto a do retalho palatino, cerca de $76 \%$. Outra grande vantagem da primeira técnica em relação a esta última é que todo o tecido cicatriza por primeira intenção, sem deixar nenhuma área de tecido cruento. O que permite um pós-operatório muito mais confortável ${ }^{12}$.

Mesmo assim, essas técnicas apresentam algumas limitações, visto que são incapazes de fechar grandes defeitos traumáticos, como os causados por projeteis de arma de fogo. Além disso, estes retalhos se limitam ao fechamento da comunicação e não reparam o defeito ósseo existente $8,10-12$.

Desde que Egyedi descreveu a técnica de fechamento da fístula buco-sinusal utilizando o corpo adiposo bucal, esta se tornou amplamente utilizada para este fim. Nas últimas quatro décadas, vários autores recorreram ao uso do tecido adiposo bucal no fechamento das comunicações ${ }^{13}$. As vantagens relatadas de seu uso têm sido a boa disponibilidade do retalho do tecido, o grande aporte sanguíneo (em comparação ao retalho palatino) e a possibilidade de 
fechamento em pelo menos duas camadas. Devido a estes fatores esta técnica possui altas taxas de sucesso e raras complicações ${ }^{14,15}, 16$. As limitações que podem ser atribuídas a essa técnica são uma perda de profundidade de vestíbulo no local da cirurgia e dificuldade de execução em pacientes com índice de massa corporal muito baixo e que que apresentem pouca disponibilidade do corpo adiposo bucal'1.

Para se obter sucesso no procedimento é imperativo que se trate qualquer infecção aguda que esteja instalada no local antes do procedimento ${ }^{17,18}$. Além disso, durante a cirurgia de fechamento, é indicado que corpos estranhos, a mucosa sinusal infectada (e normalmente hiperplásica) e o trato fistuloso devam ser removidos ${ }^{18}$. Raldi reafirma que nos casos onde haja sinusite e infecção ativa, esta deve ser controlada antes do procedimento cirúrgico ${ }^{19}$. Este mesmo autor recomenda o uso 500mg de amoxicilina (podendo ser associada ao clavulonato de potássio para maximizar o seu efeito) de 8 em 8 horas durante uma semana e irrigações diárias do seio maxilar através da fístula com soro fisiológico $0,9 \%$ até o dia da cirurgia para diminuição importante da contagem bacteriana ${ }^{18-20}$.

\section{Conclusão}

Assim como pudemos observar no relato em questão, pode-se concluir que o tratamento das fístulas buco-sinusais é cirúrgico e que a técnica de retalho bucal com o tecido adiposo bucal é uma técnica efetiva, segura, de baixa morbidade, baixo custo e com bons resultados clínicos.

\section{Referências}

1. Abuabara A, Cortez A, Passeri L, Moraes MD, Moreira R. Evaluation of different treatments for oroantral/oronasal communications: experience of 112 cases. International Journal of Oral and Maxillofacial Surgery. 2006; 35(2):155-8.

2. Emes Y, Aga U, Cesur A, Soluk-Tekkesin M, Aybar B, Alatli C. Primary Closure of Oroantral Communication Using Pedicled Buccal Fat Pad Following Maxillary Cyst Enucleation. Journal of Craniofacial Surgery. 2018; 29(2):131-133.

3. Demetoglu U, Ocak H, Bilge S. Closure of Oroantral Communication With Plasma-Rich Fibrin Membrane. Journal of Craniofacial Surgery. 2018; 29(4):367-370.

4. Kapustecki M, Niedzielska I, Marek HB, Rożanowski B. Alternative method to treat oroantral communication and fistula with autogenous bone graft and platelet rich fibrin. Med Oral Patol Oral y Cir Bucal. 2016; 21(5):608-613.

5. Daif ET. Long-Term Effectiveness of the Pedicled Buccal Fat Pad in the Closure of a Large Oroantral Fistula. Journal of Oral and Maxillofacial Surgery. 2016; 74(9):1718-1722. 
6. Franco-Carro B, Barona-Dorado C, Martinez-Gonzalez M, Rubio-Alonso L, Martinez-Gonzalez $\mathrm{J}$. Meta-analytic study on the frequency and treatment of oral antral communications. Medicina Oral Patología Oral y Cirugia Bucal. 2011; 16(5):682-687.

7. Candamourty R, Jain M, Sankar K, Babu MR. Double-layered closure of oroantral fistula using buccal fat pad and buccal advancement flap. Journal of Natural Science, Biology and Medicine. 2012; 3(2):203-205.

8.George E. Triple-Layered Closure of an Oroantral Fistula: A Case Report. The International Journal of Oral \& Maxillofacial Implants. 2018; 33(2):33-36.

9. Borgonovo AE. Surgical Options In Oroantral Fistula Treatment. The Open Dentistry Journal. 2012; 6(1):94-98.

10. Bilginaylar K. The Use of Platelet-Rich Fibrin for Immediate Closure of Acute Oroantral Communications: An Alternative Approach. Journal of Oral and Maxillofacial Surgery. 2018; 76(2):278-286.

11. Denes SA, Tieghi R, Elia G. The Buccal Fat Pad for Closure of Oroantral Communication. Journal of Craniofacial Surgery. 2016; 27(3):327-330.

12. Visscher SH, Roon MRV, Sluiter WJ, Minnen BV, Bos RR. Retrospective Study on the Treatment Outcome of Surgical Closure of Oroantral Communications. Journal of Oral and Maxillofacial Surgery. 2011; 69(12):2956-2961.

13. Egyedi P. Utilization of the buccal fat pad for closure of oro-antral and/or oro-nasal communications. Journal of Maxillofacial Surgery. 1977; 5(4):241-244.

14. Adams T, Taub D, Rosen M. Repair of Oroantral Communications by Use of a Combined Surgical Approach: Functional Endoscopic Surgery and Buccal Advancement Flap/Buccal Fat Pad Graft. Journal of Oral and Maxillofacial Surgery. 2015; 73(8):1452-1456.

15. Penarrocha-Oltra D, Alonso-Gonzalez R, Pellicer-Chover H, Aloy-Prosper A, PenarrochaDiago M. Closure of oroantral communication with buccal fat pad after removing bilateral failed zygomatic implants: A case report and 6-month follow-up. Journal of Clinical and Experimental Dentistry. 2015; 7(1):159-162.

16. Cordero GB, Ferrer SM, Fernández L. Odontogenic Sinusitis, Oro-antral Fistula and Surgical Repair by Bichats Fat Pad: Literature Review. Acta Otorrinolaringologica (English Edition). 2016; 67(2):107-113.

17. Procacci $P$, Alfonsi $F$, Tonelli $P$, Selvaggi F, Fabris GBM, Borgia V, et al. Surgical Treatment of Oroantral Communications. Journal of Craniofacial Surgery. 2016; 27(5):1190-1196.

18. Hernando J, Gallego L, Junquera L, Villarreal P. Oroantral communications. A retrospective analysis. Medicina Oral Patología Oral y Cirugia Bucal. 2010; 15(3):499-503.

19. Raldi F, Sá-Lima J, Moraes M, Zanotti G. Fechamento de Comunicações Buco-Sinusais Utilização de Enxerto Pediculado do Corpo Adiposo Bucal. RGO. 2006; 54(2):178-181.

20. Alonso-Gonzalez R, Penarrocha-Diago M, Penarrocha-Oltra D, Aloy-Prosper A, CamachoAlonso F, Penarrocha-Diago M. Closure of oroantral communications with Bichat's buccal fat pad. Level of patient satisfaction. Journal of Clinical and Experimental Dentistry. 2015; 7(1):28-33. 